behavior" is also exhibited even after a "carbonic acid correction" has been applied to the data, as Kendall discovered."

Because of this behavior Kendall considers that "there must exist some general disturbing influence not yet taken into consideration," and he promises a more detailed discussion of this matter in a later paper. With this statement of Kendall's the writer finds himself in complete accord, with, however, the added conclusion ${ }^{2}$ that the "general disturbing influence" referred to is the presence of basic or saline impurities, or both, in the conductivity water, in amounts which are far from being negligible. The influences of such impurities are much more apparent when studying solutions of acids than in the case of neutral salt solutions because metathesis is larger and results in the removal of the ion to which $80 \%$ or more of the conductance of the electrolyte is due. It seems, therefore, to the writer than the above so-called "abnormal behavior" of acids should be taken as conclusive proof that the whole of the conducting impurity in ordinary conductivity water cannot in general be regarded as carbonic acid and hence that any proposal to employ equilibrium water and to calculate "exact" metathesis corrections cannot be approved as a general and primary method for obtaining true specific conductances at high dilutions. ${ }^{3}$

URBANA, ILL.

[CONTRIBUtion fRom the Laboratory of Physical, ChEMISTRY of the University OF Ilimois. PUblication No. 30.]

\title{
THE EQUIVALENT CONDUCTANCE OF ELECTROLYTES IN DILUTE AQUEOUS SOLUTION.
}

\section{THE EXTRAPOLATION OF CONDUCTIVITY DATA TO ZERO CONCEN- TRATION.}

By Enward W. Washburn. Received September 25, 1917.

Contents.-I. Introduction. 2. The Method of Kohlrausch. 3. The Method of A. A. Noyes. 4. The Equation of Kraus. 5. The Equation of Bates. 6. A New Graphical Method of Extrapolating. 7. Bates Method for Computing $\Lambda_{0}$.

r. Introduction.-In order to determine the value of $\Lambda_{\circ}$ and also the limiting value $K_{0}$ of the Mass-Action expression

1 THIS JOURNAL, 39, I3 (I9I 7). The "carbonic acid correction"" in this instance was, within the experimental error of the data employed, equal to the water correction, which it, therefore, canceled.

2 Frequently suggested in the past by various investigators.

${ }^{3}$ In footnote 1 , page 13 , of his second paper, Kendall makes the statement that "The concentrations of any other electrolytes present must be so minute that the conclusions obtained cannot, in any case, be significantly affected." It seems to the writer that such a statement fails to give sufficient weight to the fact that in many cases saline or alkaline impurities are much more effective than carbon dioxide. This is true even, for example, in the simple case of neutral salts as explained in Sec. 8 above. Cf. Kohlrausch and Holborn, Leitvermögen, p. 92 . 


$$
\frac{\alpha^{2} C}{\mathrm{I}-\alpha}=K_{E}
$$

when $C=0$, it is necessary to extrapolate the observed conductance data from the lowest concentration measured. This extrapolation has been usually carried out in the past by fitting some empirical function to the observed values over at least the lower portion of the concentration range open to measurement and then employing this function expressed either graphically or in the form of an equation) for determining $\Lambda_{\circ}$, or both $\Lambda_{\circ}$ and $K_{0}$. A comparison of the values obtained from the different functions employed, as well as a comparison of the arguments advanced by the authors of these functions in support of the values deduced by them, brings out in a rather striking manner the general untrustworthiness of most of the methods heretofore employed.

2. The Method of Kohlrausch.-For determining the value of $\Lambda_{0}$, Kohlrausch employed the following two-parameter equation: ${ }^{1}$

$$
\Lambda_{\circ}=\Lambda_{c} P C^{1 / 2}
$$

With regard to the validity of this method of extrapolation in the case of uni-univalent salts, Kohlrausch had the following to say: ${ }^{2}$

"In the case of these salts it seems to me that there can be no possible doubt as to the method which is at present the most probable one, because for these salts the above equation reproduces the experimental data between 0.0001 and a few thousandths $N$ with practically complete agreement..... . Since, therefore, the change of the conductivity with increasing dilution is reproduced with such exactness by such a simple relationship, it seems to me very probable that this relationship expresses a general law. This relationship admittedly does not agree with the conclusions that follow from the ionic theory which, indeed, leads to quite another relationship. This fact alone, however, cannot be considered as an objection to the relationship because there can no longer be any doubt that the consequences of the ionic theory are applicable only to weak electrolytes. The conductivity of strong electrolytes is determined by other phenomena in the solution, among which possibly polymerization is the most important. It is, of course, unfortunate that the theory does not take account of these relationships but this cannot at present be helped. At the present time I believe that the above empirical relationship constitutes the only basis upon which extrapolation can be made. I conclude, therefore, that this is the method which should be employed." (The italics are mine.)

By modifying the above function slightly so as to give the three-parameter equation

$$
\Lambda_{\circ}=A \Lambda_{c}+\Lambda_{c}^{p} C^{1 / 2}
$$

Kohlrausch found (not unnaturally) that he was able to fit the new equation to the observations up to concentrations as high as O.I $N$. The $\Lambda_{\circ}$ value obtained from this new function was practically identical with that given by the first function and Kohlrausch expresses the opinion that by employing the second function we have a means of calculating $\Lambda_{\circ}$ values

1 Kohlrausch, "Gesammelte Abhandlungen," 2, I I27, I I3I, I 132.

${ }^{2}$ Loc. cit., pp. II3I, I132. 
from the data for more concentrated solutions, thus avoiding entirely the necessity of measuring dilute solutions. ${ }^{1}$

3. The Method of A. A. Noyes.-The graphical method of extrapolation employed by A. A. Noyes and his associates ${ }^{2}$ resembles Kohlrausch's method insofar as it involves the assumption that strong electrolytes do not obey the Mass-Action law even at high dilutions. In fact, as Bates has pointed out, ${ }^{3}$ in this method the value of $K_{E}$ is implicitly assumed to decrease without limit and to approach zero when $C$ becomes zero.

Now the theoretical basis for the Mass-Action law is so sound that any method which denies the validity of this law as a limiting condition for all electrolytes must, it seems to the writer, be henceforth rejected on this ground alone, if for no other reason. Contrary to the above statement of Kohlrausch there has never been any real evidence presented which would require the conclusion that the ionization equilibrium of strong electrolytes follows a law which is inconsistent with the Mass-Action law at high dilutions. Of course it is true ${ }^{4}$ that if one starts with the $a$ priori assumption that the Mass-Action law will never be obeyed and then proceeds to derive a $A_{\circ}$ value in harmony with this assumption then naturally it might not be difficult with the aid of this $\Lambda_{0}$ to compute values for the Mass-Action expression $K_{E}$, which would not exhibit any pronounced tendency to conform to the Mass-Action law, but such a behavior should scarcely be considered as evidence in support of such a view as that of Kohlrausch, quoted above.

4. The Equation of Kraus.-A four-parameter empirical equation of the form

$$
\frac{\Lambda_{c}^{2} C}{\Lambda_{0}\left(\Lambda_{0}-\Lambda_{c}\right)}=K_{E}=K_{0}+k\left(C \frac{\Lambda_{c}}{\Lambda_{0}}\right)^{h}
$$

where $\Lambda_{C}$ is the "corrected" ( $i . e$. , for viscosity) equivalent conductance, has been proposed by Kraus as a general relation for expressing the dependence of conductance upon concentration. In a careful and exhaustive study of all the conductivity data available in the literature, Kraus and Bray $^{5}$ have shown that this equation, with but few exceptions, is generally applicable to all such data. An important exception occurs in the case of aqueous solutions of strong electrolytes. Using the data of Kohlrausch and Maltby for potassium chloride, Kraus and Bray state that

"With the exception of the point at 3 normal the calculated values check the experimental values within $0.1 \%$ which is the limit of the experimental error. ${ }^{6}$ Beyond

$1 \mathrm{Cf}$. the similar reasoning of Kraus and Bray referred to below in footnote 3, p. I 26 .

2 See Noyes and Falk, This JourNal, 34, 462 (IgI2).

3 THIS JOURNAL, 35, 522 (1913).

4 As Wegscheider has pointed out (Z. physik. Chem., 69, 62 I (I909)).

5 This Journal, 35, I315 (1915).

6 This statement is not strictly correct as the data of Kohlrausch and Maltby are considerably more accurate than $0.1 \%$. 
0.00 I $N$ the conductances calculated by means of the equation are smaller than those determined experimentally by Kohlrausch and Maltby."

Because of the failure of their equation to fit the observed data in the lower portion of the concentration range, Kraus and Bray question the validity of these data. The $\Lambda_{\circ}$ value I 28.3 which they obtain by disreregarding these data is consequently quite different from that, I30.0, obtained by the Kohlrausch method from the same experimental data. As arguments in favor of the validity of their conclusions, Kraus and Bray make the following statements: ${ }^{1}$

"There is always a possibility that the discrepancy in these dilute solutions is due to experimental error, such as uncertainties in the correction for the conductance of the solvent. An increase of $10 \%$ in the correction for the solvent would bring the conductances between $0.001 N$ and $0.000 \mathrm{I} N$ into agreement with Equation 3. It should be borne in mind that in these dilute solutions we have a highly complex equilibrium between the solute, the solvent and its ions, and an unknown constituent which, in part, probably consists of carbonic acid. Taking all these factors into account, it may well be expected that the corrections made for the solvent are in error by as much as 10\%. It certainly would be an unjustifiable procedure to assume, after showing that a certain dilution law holds true from 3 normal to 0.001 normal within the limits of experimental error, that the law suddenly undergoes an enormous change after reaching a dilution where there is good reason, from a theoretical standpoint, for believing that the law must hold.

"It should not be forgotten that the general equation has been found to apply to an enormous number of solutions over extreme ranges of concentration in other solvents than water, including the alcohols, which are closely related to water. Moreover, this equation admits of a rational theoretical interpretation in dilute solutions. Further, many electrolytes in water, namely, the organic acids and bases, and mercuric chloride, a weakly dissociated salt, all obey the Mass-Action law in water. To cast aside all this mass of positive evidence because of results obtained for strong electrolytes in water at concentrations below o.00I $N$ would be an irrational procedure."

Except for the important difference that the Kraus equation reduces to the Mass-Action law at high dilutions, there is a striking similarity between the method of reasoning followed by Kraus and Bray and that followed by Kohlrausch. In both instances the fact that the equation fits the data over the concentration range involved is taken as evidence supporting the conclusion that the extrapolated value of $\Lambda_{\circ}$ to which it leads must be the correct one. It seems to the writer however that the course of reasoning followed by Kraus and Bray must be condemned as illogical at almost every point, for the following reasons:

In the first place, their calculated conductance values below $0.00 \mathrm{I} N$ are lower than the observed values, a fact which they ascribe to "a highly complex equilibrium between the solute, the solvent and its ions, and an unknown constituent, which in part, probably consists of carbonic acid." Now as explained in the first paper of this series, any metatheses which could possibly take place between the salt ions and any other ions which

1 This JOURNAL, 35, 1413 (I915). 
might be present in the conductivity water would always and necessarily result in making the ordinary "water corrected" values for the salt conductivity too small, instead of too large, and whatever might be the magnitude of this error the true conductance values must necessarily be larger than those given by Kohlrausch and Maltby, and must therefore be even farther away from the "calculated values" obtained by Kraus and Bray. In other words no possible error in the "water correction" could account for the discrepancy between the observed values and those calculated from the Kraus equation. ${ }^{1}$

Secondly, the fact that their equation holds fairly well within the concentration range, $3 N$ to $0.00 \mathrm{I} N$, is, it seems to the writer, no reason at all, as they assume (and as Kohlrausch similarly assumed for his function) for concluding that it must hold below $0.00 \mathrm{I} . N$. There must indeed be a great many functions ${ }^{2}$ which could be fitted to any desired portion of the actual conductivity curve with any desired degree of exactness but which would deviate from the course of this curve in other portions and which would yield a great variety of different $\Lambda_{0}$ and $K_{0}$ values. $^{3}$

Thirdly, the assumption made by Kraus and Bray that there is good reason from a theoretical standpoint for believing that their equation must hold at high dilutions is also wholly unsupported by any evidence and, in the opinion of the writer, is entirely without justification, as will be explained further below.

5. The Equation of Bates.--In endeavoring to find, for interpolation purposes, a function which would satisfactorily express the relation between conductivity and concentration, Bates discovered that the following mathematical form was capable of expressing the conductivity data of Kohlrausch and Maltby for potassium chloride between the concentrations r.o $N$ and o.ooor $N$ with great exactness, much more accurately in fact than was the case with the equation of Kraus. ${ }^{4}$

1. Unless indeed one wished to advance a purely ad hoc hypothesis to the effect that the conducting power of the carbonic acid is considerably increased by the simple presence of the salt in the solution, an hypothesis contrary to the accepted facts concerning the influence of small amounts of strong electrolytes upon the ionization of weak ones.

2 Cf. Wegscheider, Loc. cit., p. 622.

8 The error in logic involved in an argument of this kind is a very common one and has been made in the past by many investigators (the writer among others). It seems, therefore, necessary to emphasize the fact that in general, values obtained by extrapolation from a purely empirical equation carry no assurance whatever of being correct, the uncertainty being the greater the longer the extrapolation involved and the larger the number of parameters required in the empirical equation employed. In the remark by Kraus and Bray, that, "For a more accurate determination of $\Lambda_{0}$, accurate conductance and viscosity data should be available between $I$ and 5 normal," (Loc. cit., p. I4I2, footnote 3 . The italics are mine), the above fallacious argument seems to the writer to have attained its height of absurdity.

"See Washburn. "Principles of Physical Chemistry," I915, p. 215, McGraw-Hill Book Co., N. Y.

The Bates equation is a three-parameter equation since the value of $\Lambda_{0}$ employed 


$$
\log \frac{\Lambda_{c}^{2} C}{\Lambda_{0}\left(\Lambda_{\circ}-\Lambda_{c}\right)}=\log K_{E}=\log K_{\circ}^{\prime}+k^{\prime}\left(C \frac{\Lambda_{c}}{\Lambda_{0}}\right)^{h^{\prime}}
$$

In other words, if the above arguments advanced by Kraus and Bray in support of their equation and the value of $\Lambda_{\circ}$ to which it leads had any validity whatever, these arguments would all apply with much greater force to Bates' equation which employs a decidedly different value of $\Lambda_{0}$. A comparison of the accuracies with which the three functions of Kohlrausch, of Kraus, and of Bates, respectively, are capable of expressing the experimental data is shown in Fig. 3.

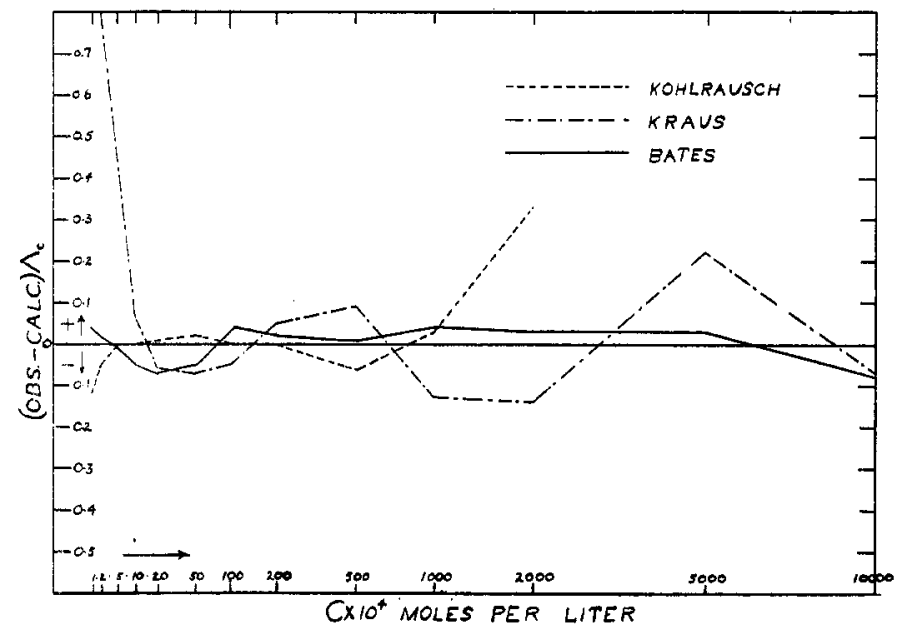

Fig. 3.-Illustrating the difference between the observed and calculated equivalent conductances of potassium chloride solutions using the empirical functions of Kohlrausch, Kraus, and Bates, respectively. The ordinates represent units of equivalent conductance.

Because the equation of Bates fits the experimental data down to the lowest concentrations heretofore measured, and with such a high degree of accuracy, most of the arguments employed by Kraus and Bray would lead to the conclusion that Bates' equation therefore represents the correct law connecting concentration and conductivity between zero and I.o $N$ and that hence the value of $K_{0}{ }^{\prime}$ in the Bates equation represents the true value of the limit approached by the Mass-Action expression as $C$ approaches zero. Such a conclusion as this, however, would be wholly unjustified. The values ( 0.08 and 0.0039 , respectively) of $K_{0}$ and $K_{\circ}{ }^{\prime}$ in the Kraus and in the Bates equations, respectively, which on the basis of

is not obtained by extrapolating the function itself but is obtained quite independently by an entirely different method which will be referred to below. Furthermore, Bates correctly regards his equation merely as an empirical interpolation equation for the concentration range in which it applies and does not ascribe any theoretical significance to any of its parameters. 
the above point of view represent the same quantity, are evidently very far from being identical and in the writer's opinion there would be no justification whatever for concluding that either one of them represents the correct value of the limit of $K_{E}$ when $C=0$.

It will be noted that the forms of both the Kraus and the Bates equations fulfill the condition of obedience to the Mass-Action law when $C=0$, and because of this fact Kraus and Bray seem inclined to attach to their own equation some theoretical significance. The mere fact that the Bates equation would, from such a standpoint, have an equally good theoretical basis is alone sufficient to destroy the force of their reasoning. Each equation imposes upon the electrolyte an arbitrary method of approach to the condition of constancy required by the Mass-Action law at high dilutions. The two methods of approach are both radically different and naturally both wrong since there is no reason whatever for supposing that either function represents the data between $C=0$ and $C$ equals the lowest concentration measured. In fact, any method of extrapolation which imposes an arbitrary a priori determined path over which the values of $K_{E}$ shall approach constancy must obviously be rejected if we are to expect to obtain from it reliable values for $\Lambda_{0}$ and $K_{0}$.

6. A New Graphical Method of Extrapolating.-In order to avoid the errors involved in what may be called the "arbitrary function" methods of extrapolating, and also to avoid the similar uncertainties involved in attempting a direct graphical extrapolation, the following method has been devised: It seems to the writer that no valid objection ${ }^{1}$ can be urged against it. It rests only upon the following two assumptions: (I) That as $C$ decreases, the value of the Mass Action expression $K_{E}$ also decreases and will eventually gradually approach a constant, $K_{0}$, at extreme dilutions; and (2) That whatever be the true form of the curve connecting $K_{E}$ with $C$ it must be one which does not show deviations from the MassAction law which increase with dilution, that is, in dilute solutions the curve must not exhibit any erratic behavior such as a sudden rise or a

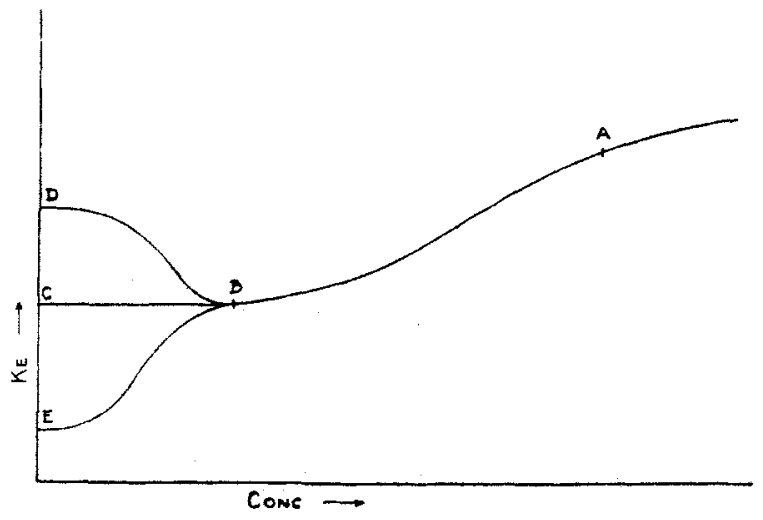

Fig. 4 .

i Unless indeed the assumption that, $\lim \mathrm{C}=\mathrm{o} d K_{E} / d \mathrm{C}=0$, is considered an objection 
sudden fall with decreasing concentration. For example, if the values of $K_{E}$ are found to follow the path $\mathrm{AB}$ (Fig. 4) to some low concentration B, it is assumed that they cannot thereafter describe such a path as $\mathrm{BD}$ or $\mathrm{BE}$, but must instead follow the path $\mathrm{BC}$.

In other words, it is assumed that those influences which cause a strong electrolyte to deviate from the Mass-Action law at high concentrations gradually and steadily become smaller and smaller and finally disappear at infinite dilution. No assumption is made as to an actual path over which the values of $K_{E}$ shall approach the constant limiting value, nor in fact is any assumption whatever made as to the mathematical form of the function which connects the conductivity with the concentration over any part of the concentration range open to direct measurement.

The new method consists simply in plotting values of $K_{E}$, the MassAction expression, against corresponding values of the concentration, employing different assumed values of $\Lambda_{\circ}$ and rejecting those values which cause the curve in dilute solutions to exhibit radical changes in direction.

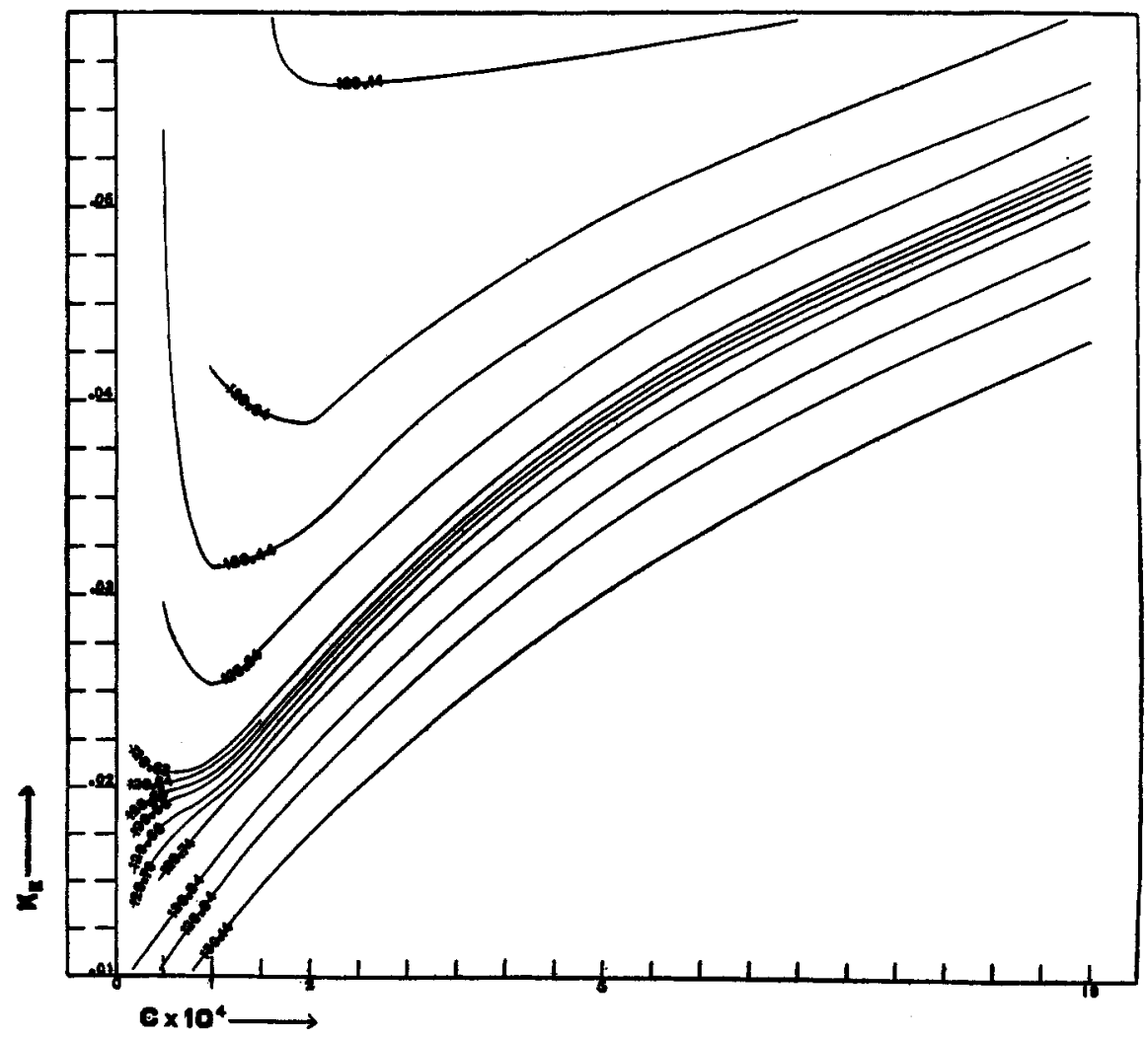

Fig. 5.-Illustrating a new method for determining $\Lambda_{0}$. 
In this way one finds that, if the value of $\Lambda_{\circ}$ employed be too small, the curve will eventually shoot upward in the lower concentration ranges, while if the $\Lambda_{\circ}$ value employed be too large the curve will turn downward in the lower concentration ranges. By employing this method it is possible to determine the value of $\Lambda_{\circ}$ with a precision of $0.01 \%$, provided the conductance data themselves are as accurate as this and extend at least as low as $C=00002 N$. This will become evident from an inspection of Fig. 5 which has been constructed from the conductance values obtained by Kohlrausch and Maltby and by Weiland, and which are tabulated in Table VII, page 146. For conductance data which only go as far as $0.000 \mathrm{I}$ $N$ this method enables one to determine the $\Lambda_{\circ}$ value to about $0.2 \%$.

7. Bates' Method for Computing $\Lambda_{0}$,--The best method heretofore suggested for determining $\Lambda_{\circ}$ values is that devised by Bates. ${ }^{1}$ This method is in a way an "arbitrary function" method since it employs the Storch equation, but as a matter of fact the uncertainties characteristic of "arbitrary function" methods in general happen to be largely eliminated in this particular method because in the process of applying the "arbitrary function" to the conductance curve it is only fitted to a small piece of the curve at a time, with the result that its two parameters, which are allowed to vary in different portions of the curve, are sufficient to secure a fairly accurate fit. There must also obviously be other functions than the Storch equation, which if carefully handled in this way could be similarly made to give reliable $\Lambda_{\circ}$ values.

In Bates' method the Storch equation

$$
\frac{\Lambda_{c}^{n} C^{n-1}}{\Lambda_{0}^{n-1}\left(\Lambda_{0}-\Lambda_{c}\right)}=\text { const. }
$$

is employed to determine an upper and a lower limit, respectively, for $\Lambda_{0}$. The lower limit is obtained by putting $n=2$, and solving for $\Lambda_{\diamond}$, using the $\Lambda_{c}$ values for the two lowest concentrations measured. In other words, the Mass-Action law is assumed to hold between these two lowest concentrations.

The upper limit for $\Lambda_{\circ}$ is determined by finding by trial the largest value which can be employed for $\Lambda_{\circ}$ without causing the corresponding values of the exponent $n$ to pass through a maximum. In other words, assuming that the Storch equation correctly expresses the conductivity curve between each pair of concentrations (an arbitrary assumption, which is of course not exactly true, but very nearly so, as explained above), then the value of $n$ is so chosen that the magnitude of the deviation of the electrolyte from the Mass-Action law (which deviation is indicated by the magnitude of the exponent $2-n$ ) shall be a minimum between the two lowest concentrations measured.

1 Thrs Journal, 35, 527 (1913). 
Except for the assumption of a definite form of function, the Storch equation, the method of Bates rests upon substantially the same character of a foundation as the one proposed by the writer, being in fact an analytical method for attaining the end which the writer secures by a graphical process. As a matter of fact when both methods are applied to the data for potassium chloride they give very nearly the same result for $\Lambda_{\circ}$. The $\Lambda_{\circ}$ values given by Bates' method are on the average only about 0.05 unit lower than those obtained by the writer's method, as shown in Table VI.

[Contribution from the Laboratory of Physical Chemistry of the University of Ilimors. Publication No. 31.]

\section{THE EQUIVALENT CONDUCTANCE OF ELECTROLYTES IN DILUTE AQUEOUS SOLUTION.}

\section{A STUDY OF DILUTE SOLUTIONS OF POTASSIUM CHLORIDE. ${ }^{1}$}

By Henry J. Weiland. 1

Received September 25, 1917.

ConTENTs. - 1 . The Preparation of Ultra-Pure Conductivity Water. (a) The Work of Previous Investigators. (b) Outline of the Distillation Process Employed in This Investigation. 2. Description of Apparatus and Experimental Procedure. (a) Description of Apparatus. (b) Collecting the Ultra-Pure Conductivity Water. 3. The Conductivity of Dilute Solutions of Potassium Chloride. (a) Preparation of the Solutions. (b) Densities of the Solutions. (c) Determination of the Cell Constant. (d) The Conductivity Data. 4. The Adsorption of Potassium Chloride by Quartz. (a) The Adsorption Correction and Method of Determining It. (b) Preparation of the Quartz Fragments. (c) Determination of the Surface of the Quartz. (d) Experimental Procedure and Results. (e) Calculation of the Adsorption Correction. 5. Discussion of the Results of the Investigation. (a) The Experimental Error. (b) Consistency of the Data. (c) Calculation of $\Lambda_{0}$ and of $K_{0}$. (d) Conductance Values at Round Concentrations. (e) Potassium Chloride and the Law of Mass Action. ( $f$ ) The Interpolation Equations of Kraus and of Bates. 6. Summary.

\section{The Preparation of Ultra-Pure Conductivity Water.}

(a) The Work of Previous Investigators. - The purest water ever obtained was that prepared by Kohlrausch and Heydweiller in I894. Their process consisted in the repeated back-and-forth distillation of water between two evacuated glass vessels, one of which was provided with electrodes. After $4^{2}$ distillations of this kind Kohlrausch and Heydweiller obtained water with a minimum conductance. of $0.043 \times 10^{-6}$ reciprocal ohms at $18^{\circ}$. The quantity of water prepared in this way was but a few cubic centimeters, not a sufficient quantity to be used in measuring the conductivity of solutions of electrolytes.

The attempts of later investigators in this field have been directed toward the problem of preparing conductivity water for use in making up

${ }^{1}$ Based upon a thesis submitted to the Graduate School of the University of Illinois, by the author, in partial fulfillment of the requirements for the degree of Doctor of Philosophy in Chemistry, June, 1917. 